\title{
MULTIPLE POSITIVE SOLUTIONS OF STRUM-LIOUVILLE EQUATIONS WITH SINGULARITIES
}

\author{
ZENGGUI WANG, LISHAN LIU, AND YONGHONG WU
}

Received 20 October 2005; Accepted 8 January 2006

The existence of multiple positive solutions for Strum-Liouville boundary value problems with singularities is investigated. By applying a fixed point theorem of cone map, some existence and multiplicity results of positive solutions are derived. Our results improve and generalize those in some well-known results.

Copyright (C) 2006 Zenggui Wang et al. This is an open access article distributed under the Creative Commons Attribution License, which permits unrestricted use, distribution, and reproduction in any medium, provided the original work is properly cited.

\section{Introduction}

In this paper, we consider the following second-order Strum-Liouville boundary value problem with singularities (BVP):

$$
\begin{gathered}
\left(p(t) u^{\prime}(t)\right)^{\prime}+g(t) F(t, u)=0, \quad \forall t \in(0,1), \\
\alpha u(0)-\beta \lim _{t \rightarrow 0^{+}} p(t) u^{\prime}(t)=0 \\
\gamma u(1)-\delta \lim _{t \rightarrow 1^{-}} p(t) u^{\prime}(t)=0
\end{gathered}
$$

where $\alpha, \beta, \gamma, \delta \geq 0$, and

$$
\rho=\beta \gamma+\alpha \gamma \int_{0}^{1} \frac{d r}{p(r)}+\alpha \delta>0 .
$$

We always assume that the following hypotheses hold:

$\left(\mathrm{A}_{1}\right) p \in C([0,1],[0,+\infty])$, and $\int_{0}^{1} d t / p(t)<+\infty$;

$\left(\mathrm{A}_{2}\right) g \in L(0,1)$, and $g(s) \geq 0$, a.e. and there exists $[a, b] \subset(0,1)$, such that $0<\int_{a}^{b} g(s) d s$;

$\left(\mathrm{A}_{3}\right) F(t, u) \in C([0,1] \times[0,+\infty],[0,+\infty])$.

The BVP(1.1) arises in many different areas of applied mathematics and physics, and only its positive solution is significant in some practice. For the special case as follows with 
$p(t) \equiv 1$ and $F(t, u)=f(u), \operatorname{BVP}(1.1)$ was studied by many authors (see $[1,4-8])$. Erbe and Wang [1] investigated this problem by using norm-type cone expansion and compression theorems; Lan and Webb [4] get the existence of positive solution for BVP(1.1) by using a well-known nonzero fixed point theorem. This problem is also studied by Ma [6] by using compact operator approximation.

Recently the authors [10] investigated the Strum-Liouville equation which has singularities at 0 and 1 , the existence of one positive solution is established by applying the fixed point index theory.

However, the authors $[1,4,6,8,10]$ only investigated the existence of positive solution for BVP(1.1). Liu and $\mathrm{Li}$ [5] get the existence of multiple positive solutions in the special case as follows with $p(t) \equiv 1$ and $g(t) \equiv 1$ by using the fixed pointed theorem. When we choose $g(t) \equiv 1$, Wang et al. [11] established some nonexistence, existence, and multiplicity results for the BVP(1.1) which are based on the Schauder fixed point theorem, the method of upper and lower solutions, and the Leray-Schauder degree theory. Furthermore, Ma and Thompson [7] studied the existence of multiple positive solutions by applying bifurcation techniques. The purpose of this paper is to consider BVP $(1.1)$ in which $g(t) \in L^{1}(0,1)$ and $F(t, u)$ satisfies weaker conditions than those in $[1,4-11]$, the existence of multiple positive solutions for $\mathrm{BVP}(1.1)$ is obtained by using a fixed point theorem. Our method is different from the ones in those papers and our results are often new even when $p(t) \equiv 1, F(t, u)=f(u)$.

A map $u \in C\left([0,1], R^{+}\right) \cap C^{1}\left((0,1), R^{+}\right), p(t) u^{\prime}(t) \in C^{1}\left((0,1), R^{+}\right)$is called a positive solution of $\operatorname{BVP}(1.1)$ if $x(t)>0$, for all $t \in(0,1)$ and $x(t)$ satisfies $\operatorname{BVP}(1.1)$.

\section{Some lemmas}

We will need the following well-known result (see, e.g., [3, Theorems 2.1 and 2.2].

Lemma 2.1. Let $K$ be a cone in a Banach space $X$. Assume that $\Omega$ is a bounded open subset of $X$ with $\theta \in \Omega$ and let $A: K \cap(\bar{\Omega}) \rightarrow K$ be a completely continuous operator. If $A u \neq \lambda x$ for $x \in K \cap \partial \Omega, \lambda \geq 1$, then $i(A, K \cap \Omega, K)=1$.

Lemma 2.2. Assume that $A: K \cap(\bar{\Omega}) \rightarrow K$ is completely continuous, and there exists $B$ : $K \cap \partial \Omega \rightarrow K$ which is completely continuous, such that

(i) $\inf _{x \in \cap \partial \Omega}\|B u\|>0$;

(ii) $x-A x \neq \lambda B x$ for $x \in K \cap \partial \Omega$, and $\lambda \geq 0$,

then $i(A, K \cap \Omega, K)=0$.

We denote by $G(t, s)$ Green's function for the homogeneous boundary value problem

$$
\begin{gathered}
\left(p(t) u^{\prime}(t)\right)^{\prime}=0, \quad \forall t \in(0,1), \\
\alpha u(0)-\beta \lim _{t \rightarrow 0^{+}} p(t) u^{\prime}(t)=0, \\
\gamma u(1)-\delta \lim _{t \rightarrow 1^{-}} p(t) u^{\prime}(t)=0 .
\end{gathered}
$$


We know that $G(t, s)$ is nonnegative on $[0,1] \times[0,1]$ and is expressed by

$$
G(t, s)= \begin{cases}\frac{1}{\rho}\left(\beta+\alpha \int_{0}^{s} \frac{d r}{p(r)}\right)\left(\delta+\gamma \int_{t}^{1} \frac{d r}{p(r)}\right), & 0 \leq s \leq t \leq 1, \\ \frac{1}{\rho}\left(\beta+\alpha \int_{0}^{t} \frac{d r}{p(r)}\right)\left(\delta+\gamma \int_{s}^{1} \frac{d r}{p(r)}\right), & 0 \leq t \leq s \leq 1,\end{cases}
$$

where $\rho=\alpha \delta+\alpha \gamma \int_{0}^{1}(d r / p(r))+\beta \gamma$.

Lemma 2.3 [2]. Green's function $G(t, s)$ has the following properties:

(i) $G(t, s) \leq G(s, s) \leq(1 / \rho)\left(\beta+\alpha \int_{0}^{1}(d r / p(r))\right)\left(\delta+\gamma \int_{0}^{1}(d r / p(r))\right):=\theta<\infty$;

(ii) for all $t \in[a, b] \subset(0,1), s \in[0,1]$, there is $G(t, s) \geq \sigma G(s, s)$, where

$$
\sigma=\min \left\{\frac{\delta+\gamma \int_{b}^{1}(d r / p(r))}{\delta+\gamma \int_{0}^{1}(d r / p(r))}, \frac{\beta+\alpha \int_{0}^{a}(d r / p(r))}{\beta+\alpha \int_{0}^{1}(d r / p(r))}\right\} .
$$

It is obvious that $0<\sigma<1$. Let $X=C[0,1], \Omega_{h}=\{u \in X:\|u\| \leq h\}$ for any $h>0$, and $K=\{u \in C[0,1]: u \geq 0$, and $x(t) \geq \sigma x(\tau)$ for $t \in[a, b], \tau \in[0,1]\}$. Then $K$ is a positive cone in $X$.

Define an operator $A$ by

$$
(A u)(t)=\int_{0}^{1} G(t, s) g(s) F(s, u(s)) d s
$$

It is well known that $u \in C[0,1] \cap C^{1}(0,1)$ is a positive solution of $\operatorname{BVP}(1.1)$ if and only if $u$ is a fixed point of the operator $A$ in $K$.

Lemma 2.4. $A: K \rightarrow K$ is completely continuous.

Proof. Similar to the proof of [10, Lemma 2.1], we can prove that $A$ is a completely continuous operator.

\section{Main result}

Theorem 3.1. Assume that $\left(A_{1}\right)-\left(A_{3}\right)$ hold, if

$\left(\mathrm{H}_{1}\right)$ there exists $p \in(0,1)$ such that $0<\liminf _{u \rightarrow 0^{+}} \min _{t \in[0,1]}\left(F(t, u) / u^{p}\right) \leq+\infty$,

$\left(\mathrm{H}_{2}\right)$ there exists $q \in(0,1]$ such that $0 \leq \limsup _{u \rightarrow+\infty} \max _{t \in[0,1]}\left(F(t, u) / u^{q}\right)<+\infty$, then $B V P(1.1)$ has at least one positive solution.

Proof. Without loss of generality, we assume that there exists $\epsilon>0$ such that $x \neq A x$ for $x \in K$ with $0<\|x\|<\epsilon$, otherwise there is a fixed point in $K$ and this would complete the proof.

By virtue of $\left(\mathrm{H}_{1}\right)$, there exists $\tau>0$ and $\epsilon_{1}>0$ such that

$$
F(t, u) \geq \tau u^{p}, \quad \text { for } 0 \leq u \leq \epsilon_{1} .
$$


4 Multiple positive solutions of singular BVP

Define $B: C[0,1] \rightarrow C[0,1]$ by

$$
B u=\phi, \quad \forall u \in C[0,1]
$$

where $\phi(t) \equiv 1, \phi \in C[0,1]$. Then it is easy to verify that $B: K \cap \partial \Omega \rightarrow K$ is completely continuous and $\inf _{K \cap \partial \Omega_{r}}\|B u\|>0, \phi \in K \backslash \theta$ with $\|\phi\|=1$.

Choose

$$
\epsilon_{2}=\min \left\{\epsilon, \epsilon_{1},\left(\sigma \delta \int_{a}^{b} G(s, s) g(s) d s\right)^{1 /(1-p)}\right\}
$$

and $r \in\left(0, \epsilon_{2}\right]$; we now prove that

$$
u-A u \neq \lambda B u, \quad \text { for } u \in K \cap \partial \Omega_{r}, \lambda \geq 0 .
$$

In fact, if not, there are $\lambda_{0} \geq 0$ and $u_{0} \in K \cap \partial \Omega_{r}$ such that $u_{0}-A u_{0}=\lambda_{0} B u_{0}$. So $\lambda_{0}>0$, then we have $u_{0}=A u_{0}+\lambda_{0} B u_{0} \geq \lambda_{0} \phi$. Let $\lambda^{*}=\sup \left\{\lambda: u_{0}(s) \geq \lambda \phi(s), s \in[a, b]\right\}$, then $\lambda^{*} \in\left[\lambda_{0},+\infty\right)$ and $u_{0}(s) \geq \lambda^{*}$ for $s \in[a, b]$. So the inequality

$$
\lambda^{*} \leq u_{0}(s) \leq\left\|u_{0}\right\|=r \leq\left(\sigma \delta \int_{a}^{b} G(s, s) g(s) d s\right)^{1 /(1-p)} \quad, \quad \forall s \in[a, b] .
$$

Then if $t \in[a, b]$, we have

$$
\begin{aligned}
u_{0}(t) & =\int_{0}^{1} G(t, s) g(s) F\left(s, u_{0}(s)\right) d s+\lambda_{0} \phi(t) \\
& \geq \int_{a}^{b} G(t, s) g(s) F\left(s, u_{0}(s)\right) d s+\lambda_{0} \\
& \geq \sigma \int_{a}^{b} G(s, s) g(s) \delta\left(u_{0}(s)\right)^{p} d s+\lambda_{0} \\
& \geq \sigma \delta\left(\lambda^{*}\right)^{p} \int_{a}^{b} G(s, s) g(s) d s+\lambda_{0} \geq \lambda^{*}+\lambda_{0}
\end{aligned}
$$

which contradicts the definition of $\lambda^{*}$, hence (3.4) holds, by Lemma 2.2

$$
i\left(A, K \cap \partial \Omega_{r}, K\right)=0 .
$$

In view of $\left(\mathrm{H}_{2}\right)$, there exists $\eta>0$ and $N>0$ such that $F(t, u) \leq \eta u^{q}$, for $u \geq C_{0}$, then

$$
0 \leq F(t, u) \leq M+\eta u^{q}, \quad \text { for } u \in[0,+\infty],
$$

where $M=\max \left\{F(t, u): 0 \leq u \leq C_{0}\right\}$.

Choose sufficiently large $R>0$ such that

$$
M R^{-1}+\eta R^{q-1}<\left(\theta \int_{0}^{1} g(s) d s\right)^{-1}
$$


We will prove that

$$
A u \neq \lambda u, \quad \text { for } u \in K \cap \partial \Omega_{R}, \lambda \geq 1 .
$$

In fact, if not, there exist $u_{1} \in K \cap \partial \Omega_{R}$ and $\lambda_{1} \geq 1$ such that $A u_{1}=\lambda_{1} u_{1}$, then if $t \in[0,1]$, we have

$$
\begin{aligned}
u_{1}(t) & \leq \lambda_{1} u_{1}(t)=\int_{0}^{1} G(t, s) g(s) F\left(s, u_{1}(s)\right) d s \\
& \leq \int_{0}^{1} G(t, s) g(s)\left(M+\eta u_{1}(s)^{q}\right) d s \\
& \leq\left(M+\eta R^{q}\right) \int_{0}^{1} G(t, s) g(s) d s \\
& \leq\left(M+\eta R^{q}\right) \theta \int_{0}^{1} g(s) d s .
\end{aligned}
$$

Hence $R \leq\left(M+\eta R^{q}\right) \theta \int_{0}^{1} g(s) d s$. That is,

$$
R \leq\left(M+\eta R^{q}\right) \theta \int_{0}^{1} g(s) d s
$$

which yields

$$
M R^{-1}+\eta R^{q-1} \geq\left(\theta \int_{0}^{1} g(s) d s\right)^{-1},
$$

which is a contradiction to (3.9), so (3.10) holds. By Lemma 2.1, we have

$$
i\left(A, K \cap \partial \Omega_{R}, K\right)=1,
$$

(3.7) and (3.14) together imply

$$
i\left(A, K \cap\left(\Omega_{R} \backslash \overline{\Omega_{r}}\right), K\right)=i\left(K \cap \partial \Omega_{R}, K\right)-i\left(A, K \cap \partial \Omega_{r}, K\right)=0-1=-1 .
$$

Consequently, according to [2, Theorem 2.3.2], $A$ has a fixed point $u^{*} \in K \cap\left(\Omega_{R} \backslash \Omega_{r}\right)$, so $\operatorname{BVP}(1.1)$ has at least one positive solution $u^{*}$. This completes the proof.

Theorem 3.2. Assume that $\left(A_{1}\right)-\left(A_{3}\right)$ hold, if

$\left(\mathrm{H}_{3}\right)$ there exists $k \in(1,+\infty)$ such that $0<\limsup _{u \rightarrow+\infty} \min _{t \in[0,1]}\left(F(t, u) / u^{k}\right) \leq+\infty$,

$\left(\mathrm{H}_{4}\right)$ there exists $l \in(1,+\infty)$ such that $0 \leq \liminf _{u \rightarrow 0^{+}} \max _{t \in[0,1]}\left(F(t, u) / u^{l}\right)<+\infty$, then $B V P(1.1)$ has at least one positive solution.

Proof. By virtue of $\left(\mathrm{H}_{3}\right)$, there exist $\xi>0$ and $C_{0}>0$ such that $F(t, u) \geq \xi u^{k}$, for $u \geq C_{0}$. Choose

$$
R>\max \left\{N \sigma^{-1},\left[\min _{t \in[a, b]} \int_{a}^{b} G(t, s) g(s) d s \xi\right]^{(-1 / k-1)}\right\} .
$$


Without loss of generality, we assume that $u \neq A u$ for $u \in K \cap \partial \Omega_{R}$, otherwise the conclusion holds. Define $B: C[0,1] \rightarrow C[0,1]$ by

$$
B u=\psi, \quad \forall u \in C[0,1]
$$

where $\psi(t)=\min _{t \in[a, b]} \int_{a}^{b} G(t, s) g(s) d s, \psi \in C[0,1]$. Then it is easy to verify $B: K \cap \partial \Omega \rightarrow$ $K$ is completely continuous and $\inf _{K \cap \partial \Omega_{R}}\|B u\|>0, \phi \in K \backslash \theta$ with $\|\psi\|=C, C$ is a const.

We now prove that

$$
u-A u \neq \lambda B u, \quad \text { for } u \in K \cap \partial \Omega_{R}, \lambda \geq 0 .
$$

In fact, if not, there are $\lambda_{1} \geq 0$ and $u_{1} \in K \cap \partial \Omega_{r}$ such that $u_{1}-A u_{1}=\lambda_{1} B u_{1}$. So $\lambda_{1}>0$, then we have $u_{1}=A u_{1}+\lambda_{1} B u_{1} \geq \lambda_{1} \psi$. Let $\lambda^{*}=\sup \left\{\lambda: u_{1}(s) \geq \lambda \psi(s), s \in[a, b]\right\}$, then $\lambda^{*} \in\left[\lambda_{1},+\infty\right]$ and $u_{1}(s) \geq \lambda^{*} \psi$ for $s \in[a, b]$. Then if $t \in[a, b]$, we have

$$
\begin{aligned}
u_{1}(t) & =\int_{0}^{1} G(t, s) g(s) F\left(s, u_{1}(s)\right) d s+\lambda_{1} \psi(t) \\
& \geq \int_{a}^{b} G(t, s) g(s) F\left(s, u_{1}(s)\right) d s+\lambda_{1} \psi \\
& \geq \int_{a}^{b} G(t, s) g(s) \xi\left(u_{1}(s)\right)^{p} d s+\lambda_{1} \psi \\
& \geq \xi\left(\lambda^{*} \psi\right)^{k} \min _{t \in[a, b]} \int_{a}^{b} G(t, s) g(s) d s+\lambda_{1} \psi \geq\left(\lambda^{*}+\lambda_{1}\right) \psi,
\end{aligned}
$$

which contradicts the definition of $\lambda^{*}$, hence (3.18) holds, by Lemma 2.2

$$
i\left(A, K \cap \partial \Omega_{R}, K\right)=0 .
$$

By virtue of $\left(\mathrm{H}_{4}\right)$, there are $\mu>0$ and $\epsilon>0$ such that $0 \leq F(t, u) \leq \mu u^{l}$ for $0 \leq u \leq \epsilon$. Take

$$
0 \leq r \leq \min \left\{\epsilon, R,\left(\mu \max _{t \in[0,1]} \int_{0}^{1} G(t, s) g(s) d s\right)^{-1 /(l-1)}\right\},
$$

then we now prove that

$$
A u \neq \lambda u, \quad \text { for } u \in K \cap \partial \Omega_{r}, \mu \geq 1 .
$$

In fact, if it is not true, there exist $u_{0} \in K \cap \partial \Omega_{r}$ and $\lambda_{0} \geq 1$ such that $A u_{0}=\lambda_{0} u_{0}$. Then if $t \in[0,1]$

$$
\begin{aligned}
u_{0}(t) & \leq \lambda_{0} u_{0}(t)=\int_{0}^{1} G(t, s) g(s) F\left(s, u_{0}(s)\right) d s \\
& \leq \int_{0}^{1} G(t, s) g(s) \mu\left(u_{0}(s)\right)^{l} d s \\
& \leq r^{l}\left(\mu \max _{t \in[0,1]} \int_{0}^{1} G(t, s) g(s) d s\right),
\end{aligned}
$$


that is,

$$
r \leq \mu r^{l} \max _{t \in[0,1]} G(t, s) g(s) d s
$$

which contradicts the definition of $r$, so (3.22) holds. According to Lemma 2.1,

$$
i\left(A, K \cap \partial \Omega_{r}, K\right)=1,
$$

(3.20) and (3.25) together imply

$$
i\left(A, K \cap\left(\Omega_{R} \backslash \overline{\Omega_{r}}\right), K\right)=i\left(K \cap \partial \Omega_{R}, K\right)-i\left(A, K \cap \partial \Omega_{r}, K\right)=0-1=-1 .
$$

Consequently, according to [2, Theorem 2.3.2], $A$ has a fixed point $u^{*} \in K \cap\left(\Omega_{R} \backslash \Omega_{r}\right)$, so $\operatorname{BVP}(1.1)$ has at least one positive solution $u^{*}$. This completes the proof.

Theorem 3.3. Assume that $\left(A_{1}\right)-\left(A_{3}\right)$ hold, $\left(H_{1}\right)$ and $\left(H_{3}\right)$ are satisfied. In addition if $\left(\mathrm{H}_{5}\right)$ there exists $T_{1}$, such that

$$
T_{1}>\max _{(t, u) \in[0,1] \times\left[\sigma T_{1}, T_{1}\right]} F(t, u) \max _{t \in[0,1]} \int_{0}^{1} G(t, s) g(s) d s,
$$

then $B V P(1.1)$ has at least two positive solutions.

Proof. By the proof of Theorems 3.1 and 3.2, there exist $0<r<T_{1}<R$ such that (3.7) and (3.20) hold, respectively.

We now prove that

$$
A u \neq \lambda u, \quad \text { for } u \in K \cap \partial \Omega_{T_{1}}, \lambda \geq 1 \text {. }
$$

Otherwise, there are $u_{2} \in K \cap \partial \Omega_{T_{1}}$ and $\lambda_{2} \geq 1$ such that $A u_{2}=\lambda u_{2}$. Then if $t \in[0,1]$,

$$
\begin{aligned}
u_{2}(t) & \leq \lambda_{2} u_{2}(t)=\int_{0}^{1} G(t, s) g(s) F\left(s, u_{2}(s)\right) d s \\
& \leq \max _{(t, u) \in[0,1] \times\left[\sigma T_{1}, T_{1}\right]} F(t, u) \int_{0}^{1} G(t, s) g(s) d s \\
& \leq \max _{(t, u) \in[0,1] \times\left[\sigma T_{1}, T_{1}\right]} F(t, u) \max _{t \in[0,1]} \int_{0}^{1} G(t, s) g(s) d s,
\end{aligned}
$$

that is,

$$
T_{1} \leq \max _{(t, u) \in[0,1] \times\left[\sigma T_{1}, T_{1}\right]} F(t, u) \max _{t \in[0,1]} \int_{0}^{1} G(t, s) g(s) d s,
$$


8 Multiple positive solutions of singular BVP

which contradicts with $\left(\mathrm{H}_{5}\right)$, hence (3.28) holds. According to Lemma 2.1,

$$
i\left(A, K \cap \partial \Omega_{T_{1}}, K\right)=1
$$

(3.7), (3.20), and (3.31) together imply

$$
\begin{gathered}
i\left(A, K \cap\left(\Omega_{R} \backslash \bar{\Omega}_{T_{1}}\right), K\right)=i\left(K \cap \partial \Omega_{R}, K\right)-i\left(A, K \cap \partial \Omega_{T_{1}}, K\right)=0-1=-1, \\
i\left(A, K \cap\left(\Omega_{T_{1}} \backslash \bar{\Omega}_{r}\right), K\right)=i\left(K \cap \partial \Omega_{T_{1}}, K\right)-i\left(A, K \cap \partial \Omega_{r}, K\right)=1-0=1 .
\end{gathered}
$$

Then according to [2, Theorem 2.3.2], $A$ has two fixed points $u_{1}^{*} \in K \cap\left(\Omega_{R} \backslash \Omega_{T_{1}}\right)$ and $u_{2}^{*} \in K \cap\left(\Omega_{T_{1}} \backslash \Omega_{r}\right)$, so $\operatorname{BVP}(1.1)$ has at least two positive solutions $u_{1}^{*}, u_{2}^{*}$. This completes the proof.

Theorem 3.4. Assume that $\left(A_{1}\right)-\left(A_{3}\right)$ hold, $\left(H_{2}\right)$ and $\left(H_{4}\right)$ are satisfied. In addition if

$\left(\mathrm{H}_{6}\right)$ there exists $\mathrm{T}_{2}$, such that

$$
0<T_{2}<\min _{(t, u) \in[a, b] \times\left[\sigma T_{2}, T_{2}\right]} F(t, u) \min _{t \in[a, b]} \int_{a}^{b} G(t, s) g(s) d s,
$$

then $B V P(1.1)$ has at least two positive solutions.

Proof. By the proof of Theorems 3.1 and 3.2, there exist $0<r<T_{2}<R$ such that (3.14) and (3.25) hold, respectively.

Define $B: C[0,1] \rightarrow C[0,1]$ by

$$
B u=u, \quad \forall u \in C[0,1] .
$$

Then it is easy to verify $B: K \cap \partial \Omega \rightarrow K$ is completely continuous and $\inf _{K \cap \partial \Omega_{r}}\|B u\|>0$.

We now prove that

$$
u-A u \neq \lambda B u, \quad \text { for } u \in K \cap \partial \Omega_{T_{2}}, \lambda \geq 0 .
$$

Otherwise, there are $u_{3} \in K \cap \partial \Omega_{T_{2}}$ and $\lambda_{3} \geq 1$ such that $u_{3}-A u_{3}=\lambda_{3} B u_{3}$. Then if $t \in$ $[a, b]$,

$$
\begin{aligned}
u_{3}(t) & =A u_{3}(t)+\lambda_{3} B u_{3} \\
& \geq \int_{a}^{b} G(t, s) g(s) F\left(s, u_{3}(s)\right) d s \\
& \geq \min _{(t, u) \in[a, b] \times\left[\sigma T_{2}, T_{2}\right]} F(t, u) \int_{a}^{b} G(t, s) g(s) d s \\
& \geq \min _{(t, u) \in[a, b] \times\left[\sigma T_{2}, T_{2}\right]} F(t, u) \min _{t \in[a, b]} \int_{a}^{b} G(t, s) g(s) d s,
\end{aligned}
$$

that is,

$$
T_{2}>\min _{(t, u) \in[a, b] \times\left[\sigma T_{2}, T_{2}\right]} F(t, u) \min _{t \in[a, b]} \int_{a}^{b} G(t, s) g(s) d s,
$$


which contradicts with $\left(\mathrm{H}_{6}\right)$, hence (3.35) holds. According to Lemma 2.2,

$$
i\left(A, K \cap \partial \Omega_{T_{2}}, K\right)=0,
$$

(3.14), (3.25), and (3.38) together imply

$$
\begin{aligned}
& i\left(A, K \cap\left(\Omega_{R} \backslash \overline{\Omega_{T_{2}}}\right), K\right)=i\left(K \cap \partial \Omega_{R}, K\right)-i\left(A, K \cap \partial \Omega_{T_{1}}, K\right)=1-0=1, \\
& i\left(A, K \cap\left(\Omega_{T_{2}} \backslash \overline{\Omega_{r}}\right), K\right)=i\left(K \cap \partial \Omega_{2}, K\right)-i\left(A, K \cap \partial \Omega_{r}, K\right)=0-1=-1 .
\end{aligned}
$$

Then according to [2, Theorem 2.3.2], $A$ has two fixed points $u_{1}^{*} \in K \cap\left(\Omega_{R} \backslash \Omega_{T_{2}}\right)$ and $u_{2}^{*} \in K \cap\left(\Omega_{T_{2}} \backslash \Omega_{r}\right)$, so BVP $(1.1)$ has at least two positive solutions $u_{1}^{*}, u_{2}^{*}$. This completes the proof.

Theorem 3.5. Assume that $\left(A_{1}\right)-\left(A_{3}\right)$ hold, $T_{1}<T_{2},\left(H_{1}\right),\left(H_{2}\right),\left(H_{5}\right)$, and $\left(H_{6}\right)$ are satisfied. Then $B V P(1.1)$ admits at least three positive solutions.

Proof. By the proof of Theorem 3.1, there exist $0<r<T_{1}$ and $R>T_{2}$ such that (3.7) and (3.14) hold, respectively. By Theorems 3.3 and 3.4, (3.31) and (3.38) are valid. Therefore $\operatorname{BVP}(1.1)$ has at least three positive solutions $u_{1}^{*} \in K \cap\left(\Omega_{R} \backslash \bar{\Omega}_{T_{2}}\right), u_{2}^{*} \in K \cap\left(\Omega_{T_{2}} \backslash \bar{\Omega}_{T_{1}}\right)$, $u_{3}^{*} \in K \cap\left(\Omega_{T_{1}} \backslash \bar{\Omega}_{r}\right)$. This completes the proof.

Similar to the proof of Theorem 3.5, we can get the following theorem.

Theorem 3.6. Assume that $\left(A_{1}\right)-\left(A_{3}\right)$ hold, $T_{1}>T_{2},\left(H_{3}\right),\left(H_{4}\right),\left(H_{5}\right)$, and $\left(H_{6}\right)$ are satisfied. Then $B V P(1.1)$ admits at least three positive solutions.

Remark 3.7. In fact, if $T_{1}=T_{2}=T$, the conditions of Theorems 3.5 and 3.6 both cannot ensure that $\operatorname{BVP}(1.1)$ has at least three positive solutions or even one positive solution. The reason is that the conditions $\left(\mathrm{H}_{5}\right)$ and $\left(\mathrm{H}_{6}\right)$ imply that the inequalities

$$
\begin{array}{ll}
A u \nsupseteq u, & \text { for } u \in K \cap \Omega_{T}, \\
A u \nless & \text { for } u \in K \cap \Omega_{T},
\end{array}
$$

hold, respectively, however, the latter two contradict each other.

Remark 3.8. In this paper, if $p(t)=1, F(t, u)=f(u)$, all the theorems above still hold and the results are new. Here the function $f(u)$ and the boundary conditions are more general than in $[1,6,7]$ where $f(u)$ only satisfies $\lim _{u \rightarrow 0+} f(u) / u=0($ or $\infty), \lim _{u \rightarrow \infty} f(u) / u=\infty$ (or 0 ) and only the cases $\beta=0, \delta=0$ are considered. In addition, our method is different from those methods in $[1,6,7]$.

Remark 3.9. In the proof of theorems, one of the key steps is to find the operator $B$. We note that it is more general than the ones in $[6,8-11]$. We think not only about the superlinear, sublinear cases but also the general cases. Hence, our results improve and generalize those in some well-known papers.

\section{Examples}

In this section, we provide some examples to illustrate the validity of the results established in Section 2. 
Example 4.1. Consider the following boundary value problem:

$$
\begin{gathered}
u^{\prime \prime}+\frac{1}{\sqrt{t(1-t)}}\left[\frac{1}{4}(1+t) u^{3 / 2}(t)+u^{1 / 2}(t)\right], \quad t \in(0,1), \\
u(0)=u(1)=0
\end{gathered}
$$

Conclusion. BVP (4.1) has at least two positive solutions.

Proof. Let

$$
p(t)=1, \quad g(t)=\frac{1}{\sqrt{t(1-t)}}, \quad F(t, u)=\frac{1}{4}(1+t) u^{3 / 2}(t)+u^{1 / 2}(t),
$$

and we choose $[a, b]=[1 / 4,3 / 4] \subset(0,1)$.

It is easy to verify that the conditions $\left(A_{1}\right)-\left(A_{3}\right)$ of Theorem 3.3 are satisfied. In term of (2.1) and (2.2), the corresponding Green function is

$$
\begin{gathered}
G(t, s)= \begin{cases}s(1-t), & 0 \leq s \leq t \leq 1, \\
t(1-s), & 0 \leq t \leq s \leq 1 .\end{cases} \\
\sigma=\frac{1}{4}, \quad G(s, s)=s(1-s), \quad \int_{0}^{1} \frac{d t}{\sqrt{t(1-t)}}=\pi .
\end{gathered}
$$

We first verify the conditions $\left(\mathrm{H}_{1}\right)$ and $\left(\mathrm{H}_{3}\right)$. In fact let $p=1 / 2, k=3 / 2$, we have

$$
\begin{gathered}
\lim _{u \rightarrow 0^{+}} \min _{t \in[0,1]} \frac{F(t, u)}{u^{p}}=\lim _{u \rightarrow 0^{+}} \min _{t \in[0,1]} \frac{(1 / 4)(1+t) u^{3 / 2}(t)+u(t)}{u^{1 / 2}}=\frac{1}{4}, \\
\lim _{u \rightarrow+\infty} \min _{t \in[0,1]} \frac{F(t, u)}{u^{k}}=\lim _{u \rightarrow+\infty} \min _{t \in[0,1]} \frac{(1 / 4)(1+t) u^{3 / 2}(t)+u^{1 / 2}(t)}{u^{3 / 2}}=1 .
\end{gathered}
$$

Choosing $T_{1}=4$, we have

$$
\begin{aligned}
& \max _{(t, u) \in[0,1] \times[1,4]} F(t, u) \max _{t \in[0,1]} \int_{0}^{1} G(s, s) g(s) d s \\
& \quad=\max _{(t, u) \in[0,1] \times[1,4]}\left[\frac{1}{4}(1+t) u^{3 / 2}(t)+u^{1 / 2}(t)\right] \frac{1}{8} \pi=6 \cdot \frac{1}{8} \pi=\frac{3}{4} \pi<4 .
\end{aligned}
$$

So condition $\left(\mathrm{H}_{5}\right)$ holds. Consequently by Theorem 3.3, BVP (4.1) has at least two positive solutions.

\section{Acknowledgments}

The first and second authors were supported financially by the National Natural Science Foundation of China (10471075) and the Natural Science Foundation of Shandong Province of China (Y2003A01).

\section{References}

[1] L. H. Erbe and H. Y. Wang, On the existence of positive solutions of ordinary differential equations, Proceedings of the American Mathematical Society 120 (1994), no. 3, 743-748. 
[2] D. J. Guo and V. Lakshmikantham, Nonlinear Problems in Abstract Cones, Notes and Reports in Mathematics in Science and Engineering, vol. 5, Academic Press, Massachusetts, 1988.

[3] D. J. Guo and J. X. Sun, The calculation of topological degree and its applications, Journal of Mathematical Research and Exposition 8 (1988), no. 3, 469-480 (Chinese).

[4] K. Q. Lan and J. R. L. Webb, Positive solutions of semilinear differential equations with singularities, Journal of Differential Equations 148 (1998), no. 2, 407-421.

[5] Z. L. Liu and F. Y. Li, Multiple positive solutions of nonlinear two-point boundary value problems, Journal of Mathematical Analysis and Applications 203 (1996), no. 3, 610-625.

[6] R. Y. Ma, Positive solutions of singular second order boundary value problems, Acta Mathematica Sinica. New Series 14 (1998), no. 1, suppl., 691-698.

[7] R. Y. Ma and B. Thompson, Multiplicity results for second-order two-point boundary value problems with superlinear or sublinear nonlinearities, Journal of Mathematical Analysis and Applications 303 (2005), no. 2, 726-735.

[8] A. M. Mao, S. X. Luan, and Y. H. Ding, On the existence of positive solutions for a class of singular boundary value problems, Journal of Mathematical Analysis and Applications 298 (2004), no. 1, $57-72$.

[9] J. J. Nieto, Nonlinear second-order periodic boundary value problems, Journal of Mathematical Analysis and Applications 130 (1988), no. 1, 22-29.

[10] Y. Sun, B. L. Xu, and L. S. Liu, Positive solutions of singular boundary value problems for SturmLiouville equations, Journal of Systems Science and Mathematical Sciences 25 (2005), no. 1, 6977 (Chinese).

[11] J. Y. Wang, W. J. Gao, and Z. X. Zhang, Nonexistence, existence and multiplicity results for SturmLiouville boundary value problems, Acta Mathematica Sinica 48 (2005), no. 4, 739-746 (Chinese).

Zenggui Wang: Department of Mathematics, Qufu Normal University, Qufu 273165,

Shandong, China

E-mail address:wzg1024@eyou.com

Lishan Liu: Department of Mathematics, Qufu Normal University, Qufu 273165, Shandong, China E-mail address: 1ls@mail.qfnu.edu.cn

Current address: Department of Mathematics and Statistics, Curtin University of Technology,

Perth 6845, Australia

Yonghong Wu: Department of Mathematics and Statistics, Curtin University of Technology,

Perth 6845, Australia

E-mail address: yhwu@maths.curtin.edu.au 


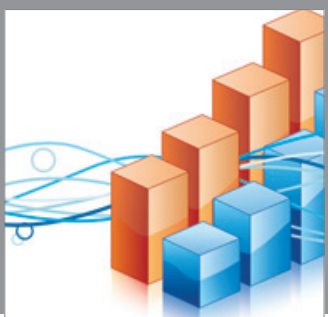

Advances in

Operations Research

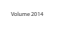

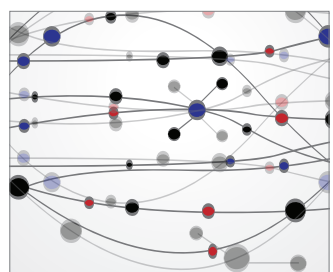

\section{The Scientific} World Journal
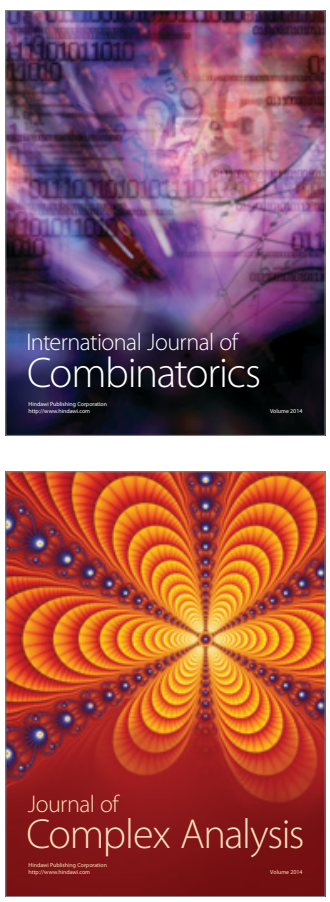

International Journal of

Mathematics and

Mathematical

Sciences
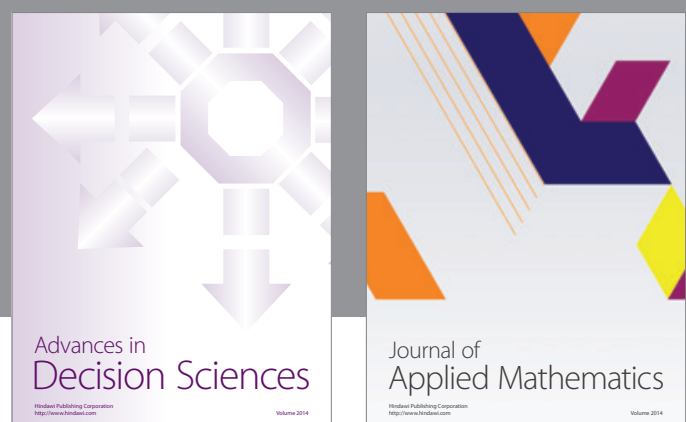

Journal of

Applied Mathematics
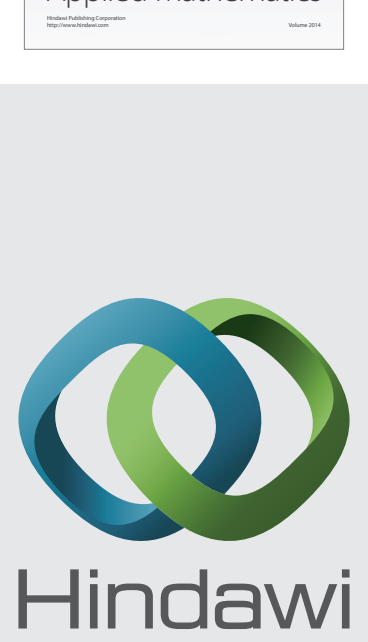

Submit your manuscripts at http://www.hindawi.com
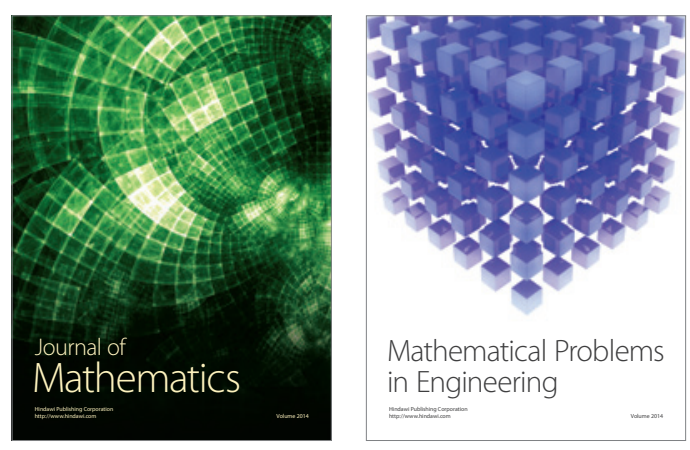

Mathematical Problems in Engineering
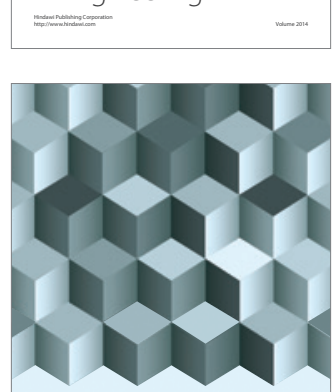

Journal of

Function Spaces
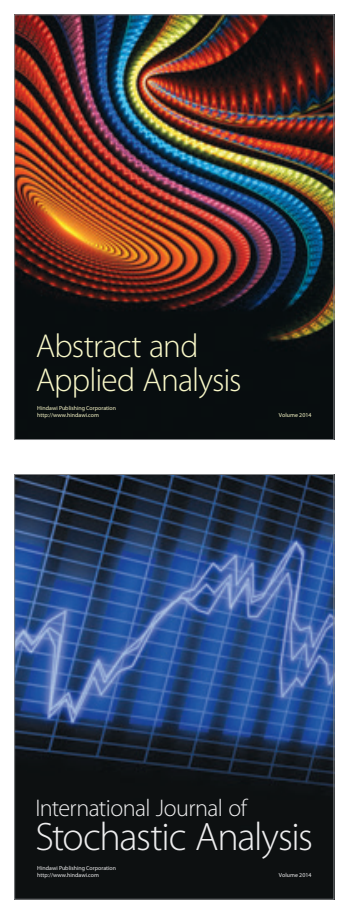

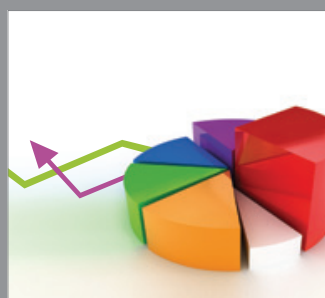

ournal of

Probability and Statistics

Promensencen
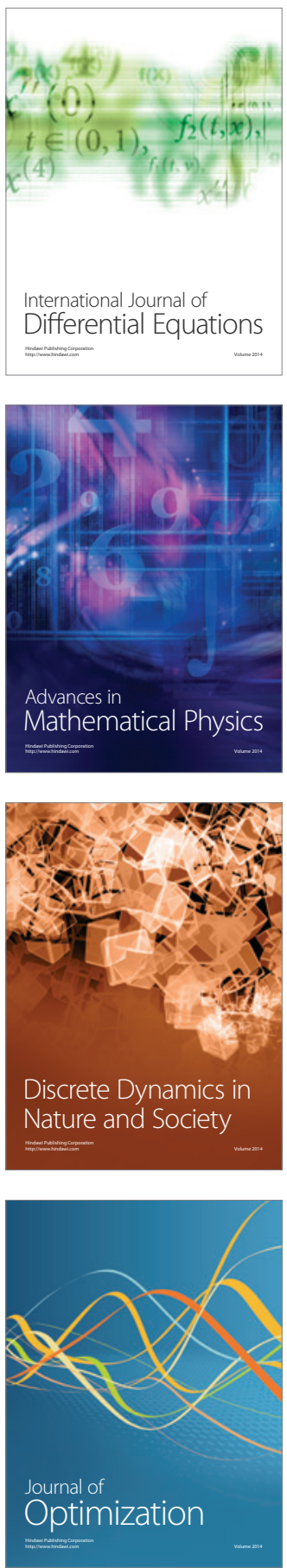